
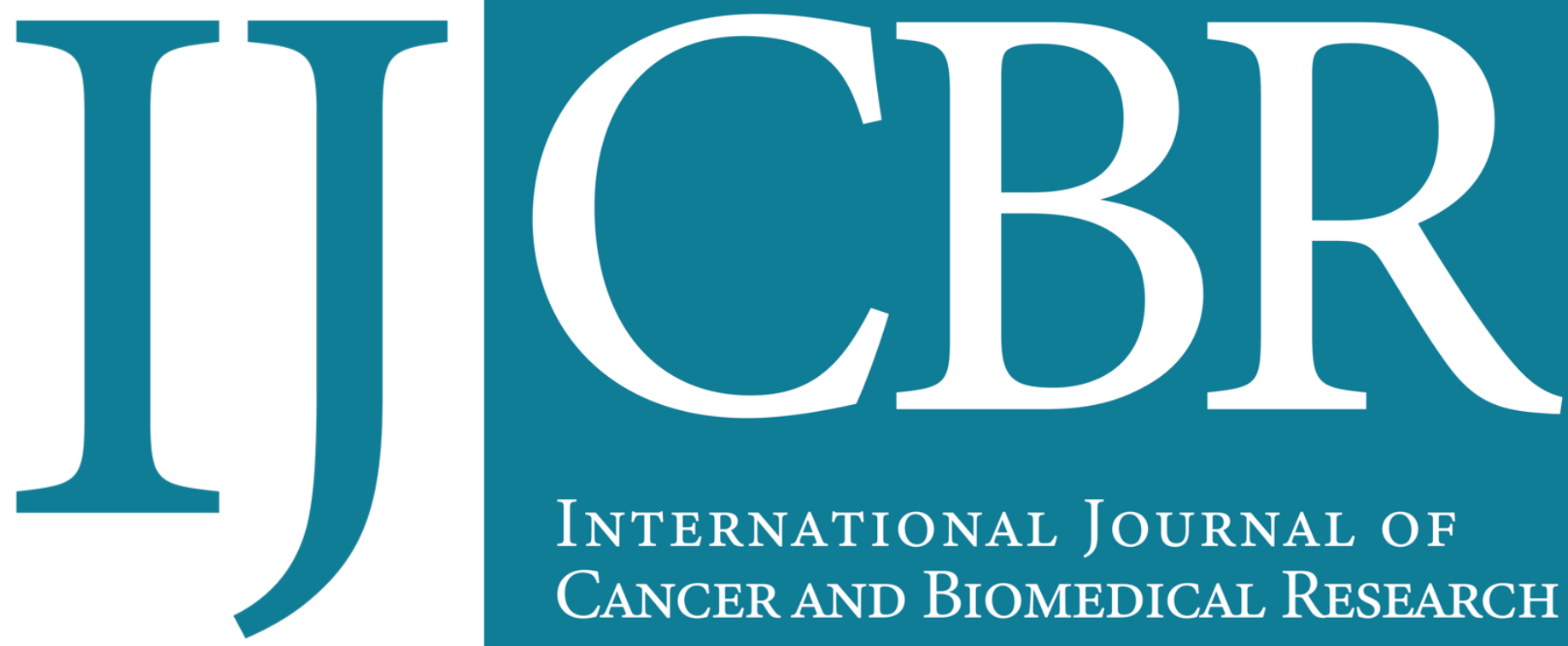

INTERNATIONAL JOURNAL OF CANCER AND BIOMEDICAL RESEARCH

https://jcbr.journals.ekb.eg

Editor-in-chief

Prof. Mohamed Labib Salem, PhD

Role of Trop2, Cyclin D1 and FOXP3 in bladder carcinoma in Egyptian patients: An immunohistochemical study

Dalia R. Al-Sharaky, Moshira M. Abdelwahed, Hend A. Kassem and Abdelnaby S. Abdelnaby 


\title{
Role of Trop2, Cyclin D1 and FOXP3 in bladder carcinoma in Egyptian patients: An immunohistochemical study
}

\author{
Dalia R. Al-Sharaky, Moshira M. Abdelwahed, Hend A. Kassem and Abdelnaby S. Abdelnaby
} Department of Pathology, Faculty of Medicine, Menoufia University, Shebin El-Kom, Egypt

Background: In Egypt, Urinary bladder carcinoma is a common malignancy accounting for $14.3 \%$ of total malignancies in both sexes with a $3: 1 \mathrm{M}$ : F ratio. To reduce bladder cancer morbidity and mortality, identification of tumor markers specific enough for prognosis and can serve as an effective anti-cancer target is urgent. The purpose of this study is to evaluate the role of Trop2, Cyclin D1, FOXP3 and their relationship with the established clinicopathological parameters and overall survival of bladder cancer patients. Methods: Using the standard immunohistochemical technique in 80 primary bladder carcinomas and 20 specimens as non-neoplastic groups. The malignant group included 50 cases of muscle-invasive and 30 cases of non-muscle invasive bladder cancer. Results: significant association of overexpressed Trop2 and FOXP3 with high grade, advanced stage, lymph node involvement, and high mitotic count. On the other hand, Cyclin D1 displayed a favorable prognostic impact and an inverse relation with Trop2 and FOXP3. A direct correlation between both FOXP3 expression in malignant cells and peritumoral TIL FOXP3+ expression was displayed. Trop2, Cyclin D1, FOXP3 expression didn't affect the overall survival of the studied sample. Conclusions: The inverse relation between Cyclin D1 and Trop2 proposes the consumption of Cyclin D1 by Trop2 as a ligand in the urinary bladder carcinogenesis. A synergistic role and a cross-talk between TIL FOXP3+ and tumoral FOXP3 + cells are anticipated. Trop2 and FOXP3 could be a promising potential biomarkers for identifying patients with poor prognostic factors in bladder cancer serving as potential targets for cancer therapy

Keywords: Trop2, Cyclin D1, FOXP3, BC, Immunohistochemistry \\ DI ARTICLE INFO}

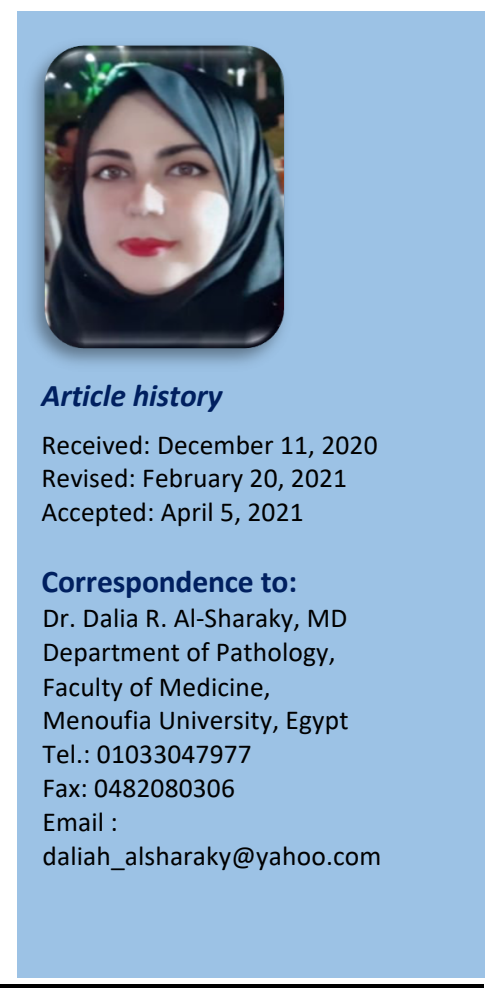

Editor-in-Chief: Prof. M.L. Salem, PhD - Article DOI: 10.21608/jcbr.2021.53189.1103

\section{INTRODUCTION}

In Egypt, Urinary bladder carcinoma is a common malignancy accounting for $14.3 \%$ of total malignancies in both sexes with 3:1 male to female ratio. It comprises $88.3 \%$ of the total urinary system tumors according to the National cancer institute registry 2016 (Ibrahim et al., 2014). The expected new cases are about 10.709 by $2020,12.762$ by 2025 and 28.337 by 2050 (Helal et al., 2015, Kyritsi et al., 2018).

To reduce bladder cancer morbidity and mortality, there is an urgent need to identify novel tumor markers which are specific enough for prognosis and can serve as effective anticancer targets (Tang et al., 2019; Heabah \& Bedeer, 2021). Trop2 is a transmembrane glycoprotein encoded by the Tacstd 2 gene
(Fong et al., 2008a), which has been actively studied as a prognostic marker and an attractive immunotherapeutic target in human cancer treatment (Guerra et al., 2013). Trop2 has several ligands, including claudin-1, claudin-7, cyclin D1, and potentially IGF-1, as for cyclin D1 is a protein encoded by CCND1 gene and it is required for progression through the G1 phase of the cell cycle (Baldin et al., 1993). Trop2 forms an oncogenic fusion protein with cyclin D1 (Huang et al., 2005). This chimera is expressed by human tumors differentially (Cubas et al., 2009). FOXP3 is a forkhead box transcription factor containing a DNA-binding domain (Lopes et al., 2006), it is known as the most specific marker of the regulatory $T$ lymphocytes (Tregs) (Fontenot et al., 2003). FOXP3 plays a crucial role in the development 
and function of Tregs, it is constitutively expressed in the nucleus of human Tregs (Martin et al., 2010).

In the scope of further understanding bladder carcinogenesis and the prognostic and predictive factors affecting it, emanates the aim of this study in investigating the role of Trop2, Cyclin D1 and FOXP3 in bladder carcinoma and correlate their expression with the available clinicopathological parameters and overall survival.

\section{METHODS}

This retrospective study included 80 primary bladder carcinoma and 20 non-neoplastic bladder specimens. The bladder carcinoma cases were received as radical cystectomy specimens ( 50 cases of muscle-invasive bladder cancer) and Transurethral Resection of Bladder Tumor (TURBT) (30 cases of non-muscle invasive bladder cancer). The 20 cases of the control group were received as cystoscopic biopsies. The cases were retrieved from the archives of Pathology Department, Faculty of Medicine, Menoufia University spanning the period between January 2017 and December 2019.

Clinical data of the studied groups: Clinical data regarding the bladder carcinoma cases were obtained from patients' medical records and documented in Table1.

Histopathological Assessment: Hematoxylin and eosin (H\&E) stained sections were evaluated for the following; Histological type according to WHO classification, 2016. Tumor grading was done according to WHO/ISUP grading criteria (Moch et al., 2016). Mitotic and apoptotic counts were counted semiquantitatively in ten randomly selected high power fields (Chauhanet al., 2016) Depth of invasion and staging of the tumor were assessed according to TNM staging system/American Joint Committee on Cancer (AJCC) Staging manual 8th edition. According to TNM classification for the stage; the malignant tumors were classified histologically as nonmuscle-invasive urinary bladder carcinoma (NMIUBC) (stage pTa and pT1) or muscleinvasive urinary bladder carcinoma (MIUBC) (stage pT2, pT3 and pT4) (Boustead et al., 2014).
Immunohistochemistry: The method used for immunostaining was streptavidin-biotin amplified system. Sections cut from the paraffin-embedded blocks were stained with Anti-Trop2 (cat\# 241308, abbexa, UK) purified rabbit poyclonal antibody was received as 0.1 $\mathrm{ml}$ conc. and diluted by phosphate buffer saline (PBS) in a dilution of 1:100. Anti-Cyclin D1 (cat\# RM-9104-R7, Thermo Fisher Scientific, USA) rabbit polyclonal antibody was received as a ready to use $7 \mathrm{ml}$ vial. Anti-FOXP3 (cat \# ARP32743, Aviva Systems Biology, USA) rabbit polyclonal antibody was received as $0.1 \mathrm{ml}$. conc. and diluted by PBS in a dilution of 1:100.

Tissue sections prepared from, normal skin as a positive control for Trop2 (Avellini et al., 2017), from normal human tonsil for Cyclin D1 (Amer and Eid 2019) and spleen for FOXP3 (18). Negative control slides were also included in each run by omitting the primary antibody.

Cytoplasmic and/or membranous staining in any number of tumor cells for Trop2 were required to assign the positivity (Avellini et al., 2017). Nuclear staining in any number of cells for Cyclin D1 was required to assign the positivity (Amer and Eid, 2019) FOXP3 was assessed in malignant epithelial tissues and the intra-tumoral and peri-tumoral infiltrating lymphocytes, cytoplasmic and nuclear staining in any number of tumor cells and tumor infiltrating lymphocytes whether (peri-tumoral or intratumoral) respectively were required to assign the positivity (Winerdal et al., 2011).

For all studied markers (Trop2, Cyclin D1 and FOXP3), $\mathrm{H}$-score system (Histochemical score): was applied to evaluate the studied section according to (Smyth et al., 2007). H score Formula $=$ strong intensity ( 3$) \times$ percentage + moderate intensity (2) $\times$ percentage + mild intensity (1) $x$ percentage. $\mathrm{H}$-score ranged from 0 to 300 and it was assessed as mean $\pm S D$, median and range.

Immunoreactivity score (IRS): was calculated depending on both intensity scoring and extent of positivity which was graded according to the German semi-quantitative scoring system as following (Godlewski et al., 2018). Intensity scoring (IS): $0=$ No staining, $1=$ Weak staining, $2=$ Moderate staining, $3=$ Strong staining. Extent of positivity was scored by percent of positive cells 
as , $0=0 \%, 1=1-10 \%$, $2=11-50 \%, 3=51-80 \%$, $4=>80 \%$. The immunoreactive score (IRS) was determined by multiplying intensity scoring by the extent of positivity ( 0 to 12 ). The cases were categorized into low and high expression scores as, Low expression Score: If the score is less than 6. High expression score: If the score is more than or equal to 6 .

Statistical Analysis: The statistical analysis was conducted using SPSS "statistical package for the social science" program for windows, version 22.0 (SPSS INC., Chicago, Illinosis, USA). Contingency tables were analyzed with descriptive statistics [Arithmetic mean $(\overline{\mathrm{x}})$, Standard deviation (SD), Percentage (\%), Median and Range] and analytic statistics[Chisquare test ( $X^{2}$ - test), Mann-Whitney $Z$ test ( $Z$ test), Kruskal-Wallis test ( $K$ test), Fisher's exact $(\mathrm{FE})$ ]. Overall survival (OS) was analyzed using the Kaplan-Meier method, and differences were examined using log-rank tests. Cox's proportional hazard regression test was used to estimate univariate and multivariate hazard ratios for prognosis. $P$ values of $\leq 0.05$ were considered statistically significant (Dawson and Trapp 2001).

\section{RESULTS}

Clinicopathologic characteristics: of primary bladder carcinoma cases (80 cases) are summarized in Table 1 , as for the clinical data of the non-neoplastic group (20) their age ranged from 30 to 77 years with a mean \pm SD of $56.8 \pm 10.9$ years with a median age of 55 years, 18 cases $(90 \%)$ were male and 2 cases $(10 \%)$ were female (Table 1$)$.

Comparison between malignant and control groups regarding Trop2, Cyclin D1 and FOXP3 immunohistochemical profile (Table 2): The expression of the studied Trop2, Cyclin D1 and FOXP3 between both nonneoplastic and UBC groups failed to reach a statistical significance. Meanwhile, membranous Trop2 was exclusively associated with the malignant group $(p=0.005)$ while patchy cytoplasmic FOXP3 was in favor of non-neoplastic group ( $p=0.011$ and 0.001 )

Relationship between Trop2, Cyclin D1 and FOXP3 IRS score and clinicopathological parameters in primary bladder carcinoma cases (Tables 3-6): Trop2 high immunoreactive score (IRS) was statistically in favor of high grade $(P=0.017)$, advanced stage $(P=0.001)$, presence of lymph node involvement $(P=0.001)$, LVI $(P=$ $0.001), P N I(P=0.005)$ and high mitotic count $(P=0.001)$ (Table 3 and Figure 1). Cyclin D1 high Immunoreactive score (IRS) was correlated with an early-stage group ( $\mathrm{P}=0.001)$ and low mitotic count $(\mathrm{P}=0.007)$. Moreover, all high IRS Cyclin D1 cases (20/20) displayed an absence of bilharzial infestation $(P=0.001)$ (Table 4 and Figure 2). Regarding tumoral FOXP3 immunoreactive score (IRS), high FOXP3 IRS was significantly in favor of advanced stage $(p=0.003)$, lymph node metastasis $(p=0.025)$, LVI and high mitotic count ( $\mathrm{P}=0.008)$ (Table 5 and Figure 3 ). Regarding the peritumoral FOXP3 expression, positive FOXP3 was statistically in favor of inflammatory stromal reaction $(P=0.001)$ and advanced stage, for 21 out of 33 TIL FOXP3 positive cases were observed in the advanced stage bladder cancer $(P=0.001)$ (Table 6). A significant direct relationship between tumoral FOXP3 $\mathrm{H}$-Score and peritumoral positively stained FOXP3+ TIL $\mathrm{H}$-score was observed in the current study $(p=0.001$ ) (Table 7).

Correlation between Cyclin D1 H-score, Trop2 and tumoral FOXP3 $\mathrm{H}$-scores in malignant cases: There was a significant inverse relationship between Cyclin D1 $\mathrm{H}$-score and both Trop2 and tumoral FOXP3 H-scores $(P=0.001$ and 0.005 respectively) (Table 7$)$.

Survival analysis: Univariate analysis of overall survival showed the bad prognostic impact of advanced pathological T stage $(P=0.003)$, nodal invasion $(P=0.006)$ and bilharzial infestation $(P=0.044)$ on patient's outcome (Table not shown). None of the studied primary antibodies Trop2, Cyclin D1, tumoral and peritumoral FOXP3 revealed a significant impact on the overall survival of the patients included in the study (Table not shown)

\section{DISCUSSION}

In the current study Trop2, was expressed in $85 \%$ of the non-neoplastic urothelium and in $97.5 \%$ of the malignant group $(P>0.05)$ in agreement with (Zhang et al., 2017; Ohmachi et al., 2006; Bignotti et al., 2010; Stepan et al., 2011). 
Table 1. Clinicopathological data of the studied bladder carcinoma cases (no. $=80$ )

\begin{tabular}{|c|c|c|c|}
\hline \multicolumn{2}{|l|}{ Variables } & \multirow{2}{*}{\multicolumn{2}{|c|}{$\begin{array}{c}\begin{array}{c}\text { Carcinoma } \\
\text { Group }\end{array} \\
\text { (No=80) }\end{array}$}} \\
\hline Age(years) & $\begin{array}{l}\text { Mean } \pm S D \\
\text { Median } \\
\text { Range }\end{array}$ & & \\
\hline & & No & $\%$ \\
\hline Gender & $\begin{array}{l}\text { Male } \\
\text { Female }\end{array}$ & $\begin{array}{l}66 \\
14\end{array}$ & $\begin{array}{l}82.5 \\
17.5\end{array}$ \\
\hline $\begin{array}{l}\text { Tumor Size } \\
\text { (50 cases) }\end{array}$ & $\begin{array}{l}\text { Mean } \pm S D \\
\text { Range } \\
\text { Median }\end{array}$ & \multicolumn{2}{|c|}{$\begin{array}{c}4.7 \pm 1.2 \\
4.7 \\
3-9\end{array}$} \\
\hline \multirow[b]{2}{*}{$\begin{array}{l}\text { WHO Histologic } \\
\text { Type (2016) }\end{array}$} & \multirow[b]{2}{*}{$\begin{array}{l}\text { Infiltrating UC } \\
\text { Noninvasive papillary } \\
\text { UC }\end{array}$} & NO & $\%$ \\
\hline & & $\begin{array}{c}76 \\
4\end{array}$ & $\begin{array}{c}95 \\
5\end{array}$ \\
\hline Grading & $\begin{array}{l}\text { Low } \\
\text { High }\end{array}$ & $\begin{array}{l}18 \\
62\end{array}$ & $\begin{array}{l}22.5 \\
77.5\end{array}$ \\
\hline $\begin{array}{l}\text { Muscularis } \\
\text { propria invasion }\end{array}$ & $\begin{array}{l}\text { NMUIBC } \\
\text { MIUBC }\end{array}$ & $\begin{array}{l}30 \\
50\end{array}$ & $\begin{array}{l}37.5 \\
62.5\end{array}$ \\
\hline Tumor stage & $\begin{array}{l}\text { Ta } \\
\text { T1 } \\
\text { T2 } \\
\text { T3 } \\
\text { T4 }\end{array}$ & $\begin{array}{c}4 \\
26 \\
18 \\
28 \\
4\end{array}$ & $\begin{array}{c}5 \\
32.5 \\
22.5 \\
35 \\
5\end{array}$ \\
\hline Stage grouping & $\begin{array}{l}\text { Early } \\
\text { Advanced }\end{array}$ & $\begin{array}{l}30 \\
50\end{array}$ & $\begin{array}{l}37.5 \\
62.5\end{array}$ \\
\hline $\begin{array}{l}\text { Lymph node } \\
\text { stage (no=50) }\end{array}$ & $\begin{array}{l}\text { N0 } \\
\text { N1 } \\
\text { N2 }\end{array}$ & $\begin{array}{l}20 \\
11 \\
19\end{array}$ & $\begin{array}{l}40 \\
22 \\
38\end{array}$ \\
\hline Bilharziasis & $\begin{array}{l}\text { Present } \\
\text { Absent }\end{array}$ & $\begin{array}{l}24 \\
56\end{array}$ & $\begin{array}{l}30 \\
70\end{array}$ \\
\hline LVI & $\begin{array}{l}\text { Present } \\
\text { Absent }\end{array}$ & $\begin{array}{l}20 \\
60\end{array}$ & $\begin{array}{l}25 \\
75\end{array}$ \\
\hline PNI & $\begin{array}{l}\text { Present } \\
\text { Absent }\end{array}$ & $\begin{array}{l}10 \\
70 \\
\end{array}$ & $\begin{array}{l}12.5 \\
87.5\end{array}$ \\
\hline Necrosis & $\begin{array}{l}\text { Present } \\
\text { Absent }\end{array}$ & $\begin{array}{l}28 \\
52\end{array}$ & $\begin{array}{l}35 \\
65\end{array}$ \\
\hline Stromal reaction & $\begin{array}{l}\text { Desmoplastic } \\
\text { Inflammatory } \\
\text { Both }\end{array}$ & $\begin{array}{l}39 \\
29 \\
13\end{array}$ & $\begin{array}{l}47.5 \\
36.3 \\
16.2\end{array}$ \\
\hline Mitosis & $\begin{array}{l}\text { Mean } \pm S D \\
\text { Range } \\
\text { Median }\end{array}$ & & $\begin{array}{l} \pm 2.6 \\
15 \\
5\end{array}$ \\
\hline Apoptosis & $\begin{array}{l}\text { Mean } \pm S D \\
\text { Range } \\
\text { Median } \\
\end{array}$ & $\begin{array}{r}15 \\
3\end{array}$ & $\begin{array}{l} \pm 6.5 \\
31 \\
4 \\
\end{array}$ \\
\hline
\end{tabular}

SD: Standard deviation, No: number, UC: Urothelial carcinoma

Transcription factors are known to be involved in cancer cell progression, such as WT1 regulate Trop2 transcription (Zaman et al., 2019), in addition, overexpression of Trop2 may be due to Trop2's intrinsic regulatory effects on cancer cell growth, invasion, and proliferation (Shyartsur and Bonavida, 2015). Overexpression of Trop2 naturally leads to tumor progression as a key driver of cancer growth (Guerra et al., 2013). On the other hand, Trop2 was found to be overexpressed across normal tissues in animal models, including the bladder, uterus, kidney, lung, and skin (Stepan et al., 2011).

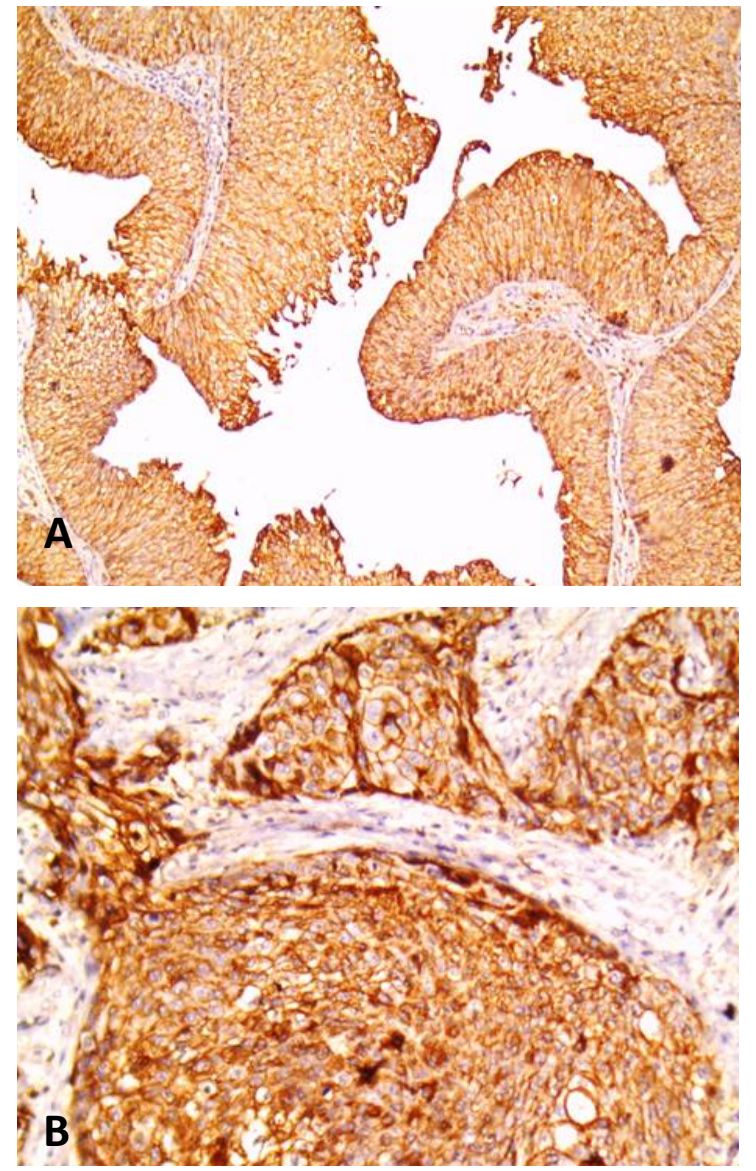

Figure 1. A): A case of non invasive papillary urothelial carcinoma showed strong membranocytoplasmic immunoreactivity of TROP2 (Immunoperoxidasex100). B): $\quad$ Strong diffuse membranocytoplasmic immunoreactivity of TROP2 in a case of muscle invasive urinary bladder carcinoma (Immunoperoxidasex200).

The expression of Trop2 in normal tissues may play an important role in normal tissue homeostasis. EpCAM, the Trop2 paralog, is thought to function as an epithelial cell adhesion molecule (Litvinov et al., 1997; Balzar et al., 2001). Trop2 shares a conserved cysteine-rich region in its extracellular domain that is required for EpCAM-mediated adhesions (Balzar et al., 2001). Trop2 high immunoreactive score (IRS) was significantly associated with poor prognostic factors as high grade, advanced stage, presence of lymph node involvement, LVI, PNI and high mitotic count. These results are in agreement with (Zhang et al., 2017) who demonstrated a significant association of overexpressed Trop2 with high tumor grade, advanced stage, and recurrence in UBC, not only in bladder cancer but also in gastric carcinoma. 

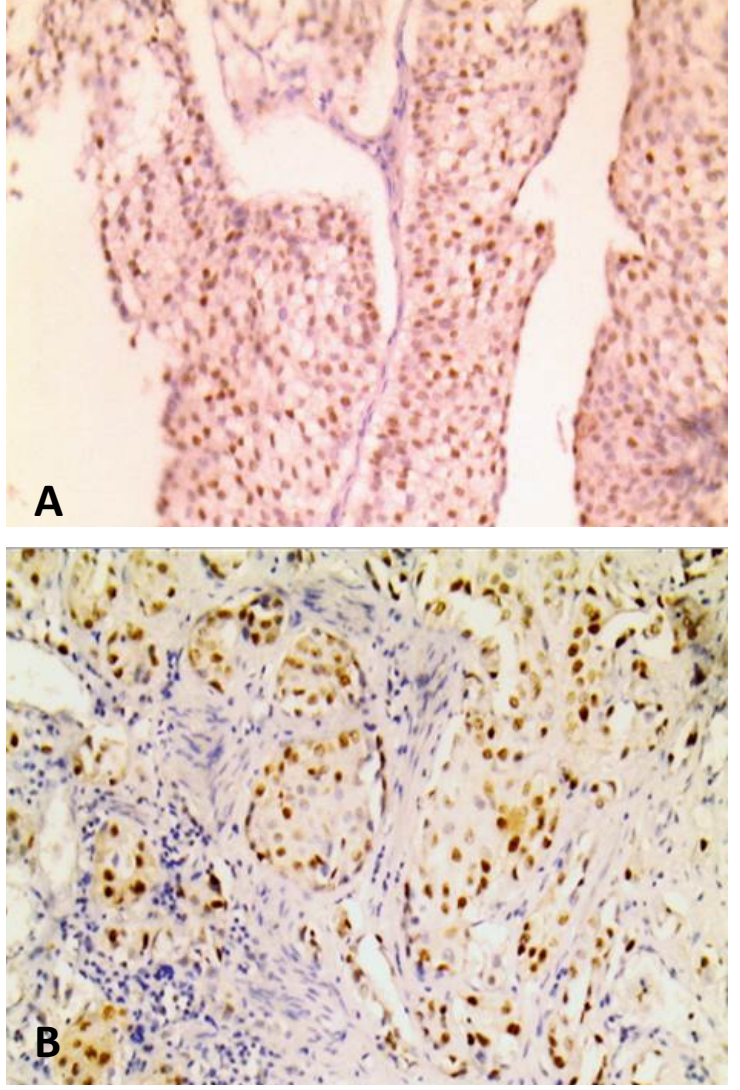

Figure 2. A): Moderate to strong nuclear Cyclin D1 immunoreactivity in a case of Non-invasive papillary lowgrade urothelial carcinoma (Immunoperoxidase x 200). B): Moderate to strong nuclear Cyclin D1 immunoreactivity in a case of muscle-invasive urinary bladder carcinoma (Immunoperoxidase x200).

Studies such as (Li et al., 2016; Li et al., 2017; Tang et al., 2019) stated that overexpression of Trop2 enhances the proliferation, migration, and invasion of malignant cells in lung cancer, gall bladder cancer and oral squamous cell carcinoma (OSCC) respectively, while downregulation of Trop2 triggered apoptosis and impaired proliferation. Activation and regulation of ERK pathway in cervical cancer cells and regulation of PI3K/AKT pathway inducing EMT in gall bladder cancer by Trop2 was stated by studies done by (Li et al., 2013; Li et al., 2017). Moreover, studies done by (Zhang et al., 2014; Redlich et al., 2018) found that loss of Trop2 leads to autocrine activation of the EGFR family member ErbB3 through neuregulin1 in the mesenchymal subtype of head and neck squamous cell cancer (HNSCC) and induced sensitivity to anti-ErbB3 antibodies, leading to reduced proliferation and tumorigenic growth in HNSCC cells.
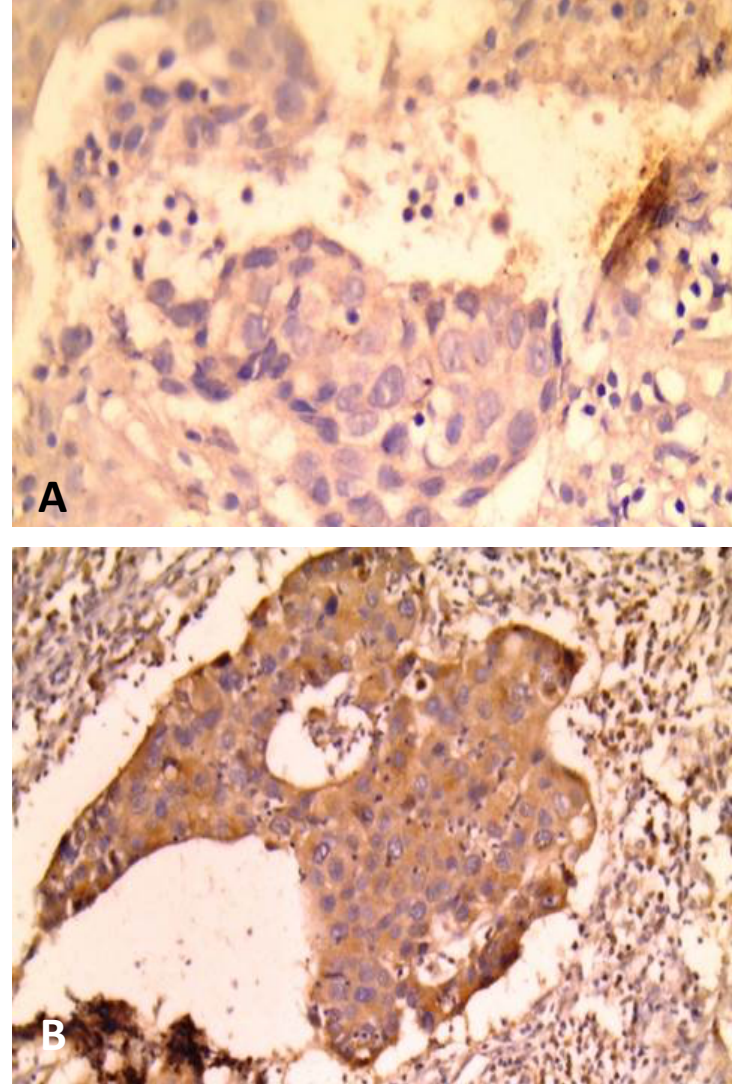

Figure 3. A): Mild cytoplasmic epithelial FOXP3 immunoreactivity with negative peritumoral lymphocytes in a case of non-muscle invasive urinary bladder (Immunoperoxidasex400). B): Moderate to strong cytoplasmic epithelial FOXP3 with moderate scattered strong nuclear peritumoral lymphocytes FOXP3 immunoreactivity in a case of muscle-invasive urinary bladder carcinoma (Immunoperoxidasex200).

Regarding Cyclin D1, it was expressed in $85 \%$ of the non-neoplastic urothelium and in $76.2 \%$ of the malignant group $(P>0.05)$, in the current study. Our results are in congruity with Khabaz et al., 2016 who reported Cyclin D1 immunoreactivity similarly frequent in bladder tumors (51.6\%) and normal tissue of bladder (50\%). A study done in 2007 by (Shariat et al., 2007) agreeing, reported uniformly intense expression of Cyclin D1in the non-neoplastic group. However other studies (Kopparapu et al., 2013) reported higher Cyclin D1 protein expression in UBC and in endometrial carcinoma (Yan et al., 2017) compared to the adjacent normal tissue. Meanwhile, other studies (Takagi et al., 2000; Mhawech et al., 2004; Fristrup et al., 2014) reported complete absence of Cyclin D1 in normal urothelium and in other tissues as colonic and gastric mucosa (Bahnassy et al., 2004; Gao et al., 2004; Shan et al., 2017) expressed in the carcinoma group. 
Table 2. Comparison between the non- neoplastic group and UBC group regarding the immunohistochemical profile of the studied TROP2, Cyclin D1 and FOXP3

\begin{tabular}{|c|c|c|c|c|c|c|c|}
\hline \multicolumn{2}{|c|}{ Variables } & \multicolumn{2}{|c|}{$\begin{array}{l}\text { Non neoplastic } \\
\text { group }(\mathrm{No}=20)\end{array}$} & \multicolumn{2}{|c|}{$\begin{array}{c}\text { Malignant group } \\
(\mathrm{No}=\mathbf{8 0})\end{array}$} & \multirow[t]{2}{*}{$\begin{array}{c}\text { Test of } \\
\text { significance }\end{array}$} & \multirow[t]{2}{*}{ P-value } \\
\hline & & No & $(\%)$ & No & $(\%)$ & & \\
\hline \multirow[t]{2}{*}{ Trop2 positivity } & $\begin{array}{l}\text { Positive } \\
\text { Negative }\end{array}$ & $\begin{array}{c}17 \\
3\end{array}$ & $\begin{array}{l}85 \\
15\end{array}$ & $\begin{array}{c}78 \\
2\end{array}$ & $\begin{array}{c}97.5 \\
2.5\end{array}$ & $\mathrm{FE}=5.6$ & 0.056 \\
\hline & & \multicolumn{2}{|c|}{$\mathrm{No}=17$} & \multicolumn{2}{|c|}{$\mathrm{No}=78$} & & \\
\hline Percentage & $\begin{array}{l}\text { Mean } \pm \text { SD } \\
\text { Range } \\
\text { Median }\end{array}$ & \multicolumn{2}{|c|}{$\begin{array}{c}64.1 \pm 15.9 \\
30-90 \\
70\end{array}$} & \multicolumn{2}{|c|}{$\begin{array}{c}72.0 \pm 19.2 \\
20-100 \\
77.5\end{array}$} & $\mathrm{FE}=1.7$ & 0.081 \\
\hline $\begin{array}{l}\text { Trop2 Subcellular } \\
\text { localization }\end{array}$ & $\begin{array}{c}\text { Membranous } \\
\text { Membranocytoplasmic }\end{array}$ & $\begin{array}{c}0 \\
17\end{array}$ & $\begin{array}{c}0 \\
100\end{array}$ & $\begin{array}{l}52 \\
26\end{array}$ & $66.7 \quad 33.3$ & $\mathrm{FE}=7.8$ & $0.005^{*}$ \\
\hline $\begin{array}{c}\text { Trop2 predominant } \\
\text { intensity }\end{array}$ & $\begin{array}{l}\text { Mild } \\
\text { Moderate } \\
\text { Strong }\end{array}$ & $\begin{array}{c}0 \\
4 \\
13\end{array}$ & $\begin{array}{c}0 \\
23.5 \\
76.5\end{array}$ & $\begin{array}{l}10 \\
27 \\
41\end{array}$ & $\begin{array}{c}12.834 .6 \\
52.6\end{array}$ & $\mathrm{FE}=4.1$ & 0.128 \\
\hline $\begin{array}{c}\text { Trop2 pattern } \\
\text { of staining }\end{array}$ & $\begin{array}{l}\text { Patchy } \\
\text { Diffuse }\end{array}$ & $\begin{array}{c}5 \\
12\end{array}$ & $\begin{array}{l}29.4 \\
70.6\end{array}$ & $\begin{array}{l}19 \\
59\end{array}$ & $\begin{array}{l}24.4 \\
75.6\end{array}$ & $\mathrm{FE}=0.189$ & 0.759 \\
\hline Trop2 IRS & $\begin{array}{l}\text { Low } \\
\text { High }\end{array}$ & $\begin{array}{c}6 \\
11\end{array}$ & $\begin{array}{l}35.3 \\
64.7\end{array}$ & $\begin{array}{l}31 \\
47\end{array}$ & $\begin{array}{l}39.7 \\
60.3\end{array}$ & $\mathrm{X}^{2}=0.116$ & 0.733 \\
\hline Trop2 H Score & $\begin{array}{l}\text { Mean } \pm \text { SD } \\
\text { Median } \\
\text { Range }\end{array}$ & \multicolumn{2}{|c|}{$\begin{array}{c}174.1 \pm 56.3 \\
50-240 \\
180\end{array}$} & \multicolumn{2}{|c|}{$\begin{array}{c}182.5 \pm 73.9 \\
20-300 \\
190\end{array}$} & $\mathrm{FE}=0.696$ & 0.487 \\
\hline Cyclin D1 expression & $\begin{array}{l}\text { Positive } \\
\text { Negative }\end{array}$ & $\begin{array}{c}17 \\
3\end{array}$ & $\begin{array}{l}85 \\
15\end{array}$ & $\begin{array}{l}61 \\
19\end{array}$ & $\begin{array}{l}76.2 \\
23.8\end{array}$ & $\mathrm{FE}=0.714$ & 0.551 \\
\hline & & \multicolumn{2}{|c|}{$\mathrm{No}=17$} & \multicolumn{2}{|c|}{$\mathrm{No}=61$} & & \\
\hline Percentage & $\begin{array}{c}\text { Mean } \pm \text { SD } \\
\text { Range } \\
\text { Median }\end{array}$ & \multicolumn{2}{|c|}{$\begin{array}{c}39.4 \pm 21.5 \\
10-80 \\
40\end{array}$} & \multicolumn{2}{|c|}{$\begin{array}{c}48.4 \pm 21.6 \\
10-90 \\
50\end{array}$} & $\mathrm{U}=1.5$ & 0.123 \\
\hline \multirow[b]{2}{*}{$\begin{array}{l}\text { Predominant } \\
\text { intensity }\end{array}$} & & $\mathrm{NO}$ & $\%$ & NO & $\%$ & \multirow[b]{2}{*}{$\mathrm{FE}=1.7$} & \multirow[b]{2}{*}{0.425} \\
\hline & $\begin{array}{l}\text { Mild } \\
\text { Moderate } \\
\text { Strong }\end{array}$ & $\begin{array}{c}2 \\
10 \\
5\end{array}$ & $\begin{array}{l}11.8 \\
58.8 \\
29.4\end{array}$ & $\begin{array}{l}10 \\
25 \\
26\end{array}$ & $\begin{array}{c}16.4 \\
41 \\
42.6\end{array}$ & & \\
\hline Cyclin D1 IRS & $\begin{array}{l}\text { Low } \\
\text { High }\end{array}$ & $\begin{array}{c}15 \\
2\end{array}$ & $\begin{array}{l}88.2 \\
11.8\end{array}$ & $\begin{array}{l}41 \\
20\end{array}$ & $\begin{array}{l}67.2 \\
32.8\end{array}$ & $\mathrm{FE}=2.9$ & 0.129 \\
\hline Distribution & $\begin{array}{l}\text { Patchy } \\
\text { Diffuse }\end{array}$ & $\begin{array}{c}11 \\
6\end{array}$ & $\begin{array}{l}64.7 \\
35.3\end{array}$ & $\begin{array}{l}43 \\
18\end{array}$ & $\begin{array}{l}70.5 \\
29.5\end{array}$ & $x^{2}=0.209$ & 0.648 \\
\hline Cyclin D1 H.Score & $\begin{array}{c}\text { Mean } \pm \text { SD } \\
\text { Range } \\
\text { Median }\end{array}$ & \multicolumn{2}{|c|}{$\begin{array}{c}88.5 \pm 58.1 \\
20-220 \\
80\end{array}$} & \multicolumn{2}{|c|}{$\begin{array}{c}111.1 \pm 59.4 \\
10-240 \\
120\end{array}$} & $\mathrm{U}=1.4$ & 0.123 \\
\hline FOXP3 Expression & $\begin{array}{l}\text { negative } \\
\text { positive }\end{array}$ & $\begin{array}{c}3 \\
17\end{array}$ & $\begin{array}{l}15 \\
85\end{array}$ & $\begin{array}{l}20 \\
60\end{array}$ & $\begin{array}{l}25 \\
75\end{array}$ & $\mathrm{FE}=0.903$ & 0.342 \\
\hline FOXP3 Percent & $\begin{array}{l}\text { Mean } \pm \text { SD } \\
\text { Range } \\
\text { Median }\end{array}$ & \multicolumn{2}{|c|}{$\begin{array}{c}42.9 \pm 18.2 \\
10-60 \\
50\end{array}$} & \multicolumn{2}{|c|}{$\begin{array}{c}40.0 \pm 21.7 \\
10-80 \\
30\end{array}$} & $\mathrm{U}=0.720$ & 0.471 \\
\hline FOXP3 Distribution & $\begin{array}{l}\text { Patchy } \\
\text { Diffuse }\end{array}$ & $\begin{array}{c}11 \\
6\end{array}$ & $\begin{array}{l}64.7 \\
35.3\end{array}$ & $\begin{array}{c}54 \\
6\end{array}$ & $\begin{array}{l}90 \\
10\end{array}$ & $\mathrm{FE}=6.44$ & $0.011^{*}$ \\
\hline FOXP3 Intensity & $\begin{array}{l}\text { Mild to moderate } \\
\text { strong }\end{array}$ & $\begin{array}{c}16 \\
1\end{array}$ & $\begin{array}{c}94.1 \\
5.9\end{array}$ & $\begin{array}{l}42 \\
18\end{array}$ & $\begin{array}{l}70 \\
30\end{array}$ & $\mathrm{FE}=5.6$ & 0.060 \\
\hline $\begin{array}{c}\text { FOXP3 Subcellular } \\
\text { localization }\end{array}$ & $\begin{array}{c}\text { cytoplasmic } \\
\text { apical cytoplasmic }\end{array}$ & $\begin{array}{l}9 \\
8\end{array}$ & $\begin{array}{l}52.9 \\
47.1\end{array}$ & $\begin{array}{c}60 \\
0\end{array}$ & $\begin{array}{c}100 \\
0\end{array}$ & $\mathrm{FE}=31.5$ & $0.001 *$ \\
\hline FOXP3 IRS & $\begin{array}{l}\text { Low } \\
\text { High }\end{array}$ & $\begin{array}{c}15 \\
2\end{array}$ & $\begin{array}{l}88.2 \\
11.8\end{array}$ & $\begin{array}{l}46 \\
14\end{array}$ & $\begin{array}{c}6.7 \\
23.3\end{array}$ & $\mathrm{FE}=1.077$ & 0.299 \\
\hline FOXP3 H score & $\begin{array}{c}\text { Mean } \pm \text { SD } \\
\text { Range } \\
\text { Median }\end{array}$ & $\begin{array}{r}69 \\
20\end{array}$ & & & $\begin{array}{l} \pm 53.7 \\
-200 \\
70\end{array}$ & $\mathrm{U}=0.351$ & 0.726 \\
\hline
\end{tabular}


Table 3. Relationship between Trop2 immunoreactive score (IRS) and studied clinico-pathological parameters in malignant cases

\begin{tabular}{|c|c|c|c|c|c|c|c|}
\hline & & \multicolumn{4}{|c|}{ TROP2 IRS } & \multirow{3}{*}{$\begin{array}{c}\text { test of } \\
\text { significant }\end{array}$} & \multirow{3}{*}{$P$ value } \\
\hline & & \multicolumn{2}{|c|}{$\begin{array}{c}\text { Low } \\
(\mathrm{no}=31)\end{array}$} & \multicolumn{2}{|c|}{$\begin{array}{c}\text { High } \\
(\mathrm{no}=47)\end{array}$} & & \\
\hline & & NO & $\%$ & NO & $\%$ & & \\
\hline \multirow[t]{2}{*}{ Gender } & Male $(\mathrm{no}=64)$ & 24 & 37.5 & 40 & 62.5 & \multirow[t]{2}{*}{$\mathrm{x}^{2}=0.749$} & \multirow[t]{2}{*}{0.387} \\
\hline & Female $($ no $=14)$ & 7 & 50 & 7 & 50 & & \\
\hline & mean \pm SD & \multirow{3}{*}{\multicolumn{2}{|c|}{$\begin{array}{c}63.2 \pm 12.1 \\
26-96 \\
65\end{array}$}} & \multirow{3}{*}{\multicolumn{2}{|c|}{$\begin{array}{c}63.4 \pm 9.3 \\
46-83 \\
64\end{array}$}} & $\mathrm{U}=0.092$ & 0.927 \\
\hline age/years & range & & & & & & \\
\hline & Median & & & & & & \\
\hline \multirow{2}{*}{$\begin{array}{c}\text { WHO } \\
\text { Histological Type }\end{array}$} & Infiltrating UC $(\mathrm{no}=75)$ & 30 & 40.5 & 45 & 59.5 & \multirow[t]{2}{*}{$\mathrm{FE}=0.731$} & \multirow[t]{2}{*}{0.694} \\
\hline & Noninvasive papillary $\mathrm{UC}(\mathrm{no}=3)$ & 1 & 33.3 & 2 & 66.7 & & \\
\hline \multirow{2}{*}{ Grade } & Low $($ no=17) & 11 & 64.7 & 6 & 35.3 & \multirow[t]{2}{*}{$\mathrm{x} 2=5.6$} & \multirow[t]{2}{*}{$0.017^{*}$} \\
\hline & $\operatorname{High}(\mathrm{no}=61)$ & 20 & 32.8 & 41 & 67.2 & & \\
\hline \multirow{2}{*}{ stage group } & Early $($ no $=28)$ & 24 & 85.7 & 4 & 14.3 & \multirow[t]{2}{*}{$x 2=38.5$} & \multirow[t]{2}{*}{$0.001^{*}$} \\
\hline & advanced $(\mathrm{no}=50)$ & 7 & 14 & 43 & 86 & & \\
\hline \multirow{2}{*}{$\begin{array}{l}\text { lymph node } \\
(\text { no=50) }\end{array}$} & Absent $(\mathrm{no}=20)$ & 4 & 20 & 16 & 80 & \multirow[t]{2}{*}{$\mathrm{FE}=39.1$} & \multirow[t]{2}{*}{$0.001 *$} \\
\hline & present $(\mathrm{no}=30)$ & 3 & 10 & 27 & 90 & & \\
\hline \multirow{2}{*}{ Bilharasis } & absent $(\mathrm{no}=55)$ & 23 & 41.8 & 32 & 58.2 & \multirow[t]{2}{*}{$\mathrm{x} 2=0.335$} & \multirow[t]{2}{*}{0.563} \\
\hline & present $(\mathrm{no}=23)$ & 8 & 34.8 & 15 & 65.2 & & \\
\hline \multirow{2}{*}{ Necrosis } & absent $(\mathrm{no}=50)$ & 19 & 38 & 31 & 62 & \multirow{2}{*}{$0.177(\mathrm{x} 2)$} & \multirow[t]{2}{*}{0.674} \\
\hline & present $(\mathrm{no}=28)$ & 12 & 42.9 & 16 & 57.1 & & \\
\hline \multirow{2}{*}{ LVI } & absent $($ no $=58)$ & 30 & 51.7 & 28 & 48.3 & $\mathrm{x} 2=13.5$ & $0.001 *$ \\
\hline & present $(\mathrm{no}=20)$ & 1 & 5 & 19 & 95 & & \\
\hline PNI & absent $(n o=68)$ & 31 & 45.6 & 37 & 54.4 & $\mathrm{FE}=7.6$ & $0.005^{*}$ \\
\hline PNI & present $(\mathrm{no}=10)$ & 0 & 0 & 10 & 100 & & \\
\hline & Desmoplastic $(\mathrm{no}=25)$ & 16 & 44.4 & 9 & 69.2 & $\mathrm{x} 2=0.809$ & 0.667 \\
\hline Stromal reaction & inflammatory $(\mathrm{no}=31)$ & 11 & 37.9 & 20 & 55.6 & & \\
\hline & both $(n o=22)$ & 4 & 30.8 & 18 & 62.1 & & \\
\hline & mean \pm SD & 14. & \pm 6.5 & 15 . & \pm 6.6 & $\mathrm{U}=1.13$ & 0.258 \\
\hline Apoptosis & range & & 30 & & & & \\
\hline & Median & & & & & & \\
\hline & mean $\pm \mathrm{SD}$ & 4.8 & 1.9 & & 2.7 & $\mathrm{U}=3.2$ & $0.001^{*}$ \\
\hline Mitosis & range & & & & 15 & & \\
\hline & Median & & & & & & \\
\hline
\end{tabular}

Table 4. Relationship between Cyclin D1 immunoreactive score (IRS) and the studied clinico-pathological parameters in malignant cases.

\begin{tabular}{|c|c|c|c|c|c|c|c|}
\hline \multirow{2}{*}{\multicolumn{2}{|c|}{ Variables }} & \multicolumn{4}{|c|}{ Cyclin D1 IRS } & \multirow{3}{*}{ Test of significance } & \multirow{3}{*}{$P$ value } \\
\hline & & \multicolumn{2}{|c|}{$\begin{array}{c}\text { Low } \\
(\mathrm{no}=41)\end{array}$} & \multicolumn{2}{|c|}{$\begin{array}{c}\text { High } \\
(\text { no=20) }\end{array}$} & & \\
\hline \multirow{3}{*}{ Gender } & & $\mathrm{NO}$ & $\%$ & $\mathrm{NO}$ & $\%$ & & \\
\hline & Male $(n o=50)$ & 35 & 67.3 & 15 & 32.7 & \multirow{2}{*}{$\mathrm{FE}=0.977$} & \multirow{2}{*}{0.323} \\
\hline & Female $($ no $=11)$ & 6 & 66.7 & 5 & 33.3 & & \\
\hline Age/years & mean $\pm \mathrm{SD}$ & \multicolumn{2}{|c|}{$64.0 \pm 8.2$} & \multicolumn{2}{|c|}{$61.4 \pm 13.1$} & $\mathrm{U}=0.347$ & 0.729 \\
\hline \multirow{2}{*}{ Grade } & Low $($ no=13) & 6 & 46.2 & 7 & 53.8 & \multirow{2}{*}{$\mathrm{FE}=3.2$} & \multirow{2}{*}{0.068} \\
\hline & High $(\mathrm{no}=48)$ & 35 & 72.9 & 13 & 27.1 & & \\
\hline \multirow{2}{*}{ stage group } & Early $(\mathrm{no}=27)$ & 11 & 40.7 & 16 & 59.3 & \multirow{2}{*}{$\mathrm{FE}=15.4$} & \multirow{2}{*}{$0.001 *$} \\
\hline & Advanced $($ no=34) & 30 & 88.2 & 4 & 11.8 & & \\
\hline \multirow{2}{*}{ lymph node $(\mathrm{No}=34)$} & Absent $(n o=14)$ & 13 & 92.6 & 1 & 7.4 & \multirow{2}{*}{\multicolumn{2}{|c|}{$\begin{array}{c}\text { Was not suitable statistically } \\
\text { due to small sample size (4) of high IRS }\end{array}$}} \\
\hline & Present $(n o=20)$ & 17 & 85 & 3 & 15 & & \\
\hline \multirow{2}{*}{ Bilharziasis } & absent $(n o=45)$ & 25 & 55.6 & 20 & 44.4 & \multirow{2}{*}{$\mathrm{FE}=0.6$} & \multirow{2}{*}{$0.001 *$} \\
\hline & present $(n o=16)$ & 16 & 100 & 0 & 0 & & \\
\hline \multirow{2}{*}{ LVI } & Absent $(n o=47)$ & 28 & 59.6 & 19 & 40.4 & \multirow{2}{*}{$\mathrm{FE}=5.6$} & \multirow{2}{*}{0.062} \\
\hline & Present $(n o=14)$ & 13 & 92.8 & 1 & 7.2 & & \\
\hline \multirow{2}{*}{ PNI } & Absent $(\mathrm{no}=56)$ & 37 & 66.1 & 19 & 33.9 & \multirow{2}{*}{$\mathrm{FE}=0.404$} & \multirow{2}{*}{0.525} \\
\hline & Present $(\mathrm{no}=5)$ & 4 & 80 & 1 & 20 & & \\
\hline \multirow{2}{*}{ Necrosis } & Absent $($ no=40) & 26 & 63.4 & 14 & 70 & \multirow{2}{*}{$\mathrm{FE}=0.256$} & \multirow{2}{*}{0.611} \\
\hline & Present $(n o=21)$ & 15 & 36.6 & 6 & 30 & & \\
\hline Apoptosis & mean $\pm \mathrm{SD}$ & \multicolumn{2}{|c|}{$15.5 \pm 6.3$} & \multicolumn{2}{|c|}{$14.5 \pm 5.8$} & $\mathrm{~K}=0.501$ & 0.617 \\
\hline Mitosis & mean $\pm \mathrm{SD}$ & \multicolumn{2}{|c|}{$6.3 \pm 2.7$} & 4.5 & 1.5 & $\mathrm{~K}=2.7$ & $0.007^{*}$ \\
\hline
\end{tabular}


Table 5. Relationship between tumoral FOXP3 IRS and the studied clinico-pathological parameters in malignant cases

\begin{tabular}{|c|c|c|c|c|c|c|c|}
\hline \multirow{2}{*}{\multicolumn{2}{|c|}{ Variables }} & \multicolumn{4}{|c|}{ FOXP3 IRS } & \multirow{2}{*}{$\begin{array}{c}\text { Test of } \\
\text { significance }\end{array}$} & \multirow[b]{2}{*}{$P$ value } \\
\hline & & \multicolumn{2}{|c|}{$\begin{array}{c}\text { Low } \\
(\mathrm{no}=46)\end{array}$} & \multicolumn{2}{|c|}{$\begin{array}{c}\text { High } \\
(\text { no }=14)\end{array}$} & & \\
\hline \multirow{2}{*}{ Gender } & \multirow{2}{*}{$\begin{array}{l}\text { Male }(\text { no }=51) \\
\text { Female }(\text { no }=9)\end{array}$} & $\begin{array}{l}\mathrm{N} \\
\mathrm{O}\end{array}$ & $\%$ & $\mathrm{NO}$ & $\%$ & \multirow[t]{2}{*}{$\mathrm{FE}=0.007$} & \multirow[t]{2}{*}{0.932} \\
\hline & & $\begin{array}{c}39 \\
7\end{array}$ & $\begin{array}{l}76.5 \\
77.8\end{array}$ & $\begin{array}{c}12 \\
2\end{array}$ & $\begin{array}{l}23.5 \\
22.2\end{array}$ & & \\
\hline age/years & $\begin{array}{l}\text { mean } \pm \mathrm{SD} \\
\text { range } \\
\text { Median }\end{array}$ & \multicolumn{2}{|c|}{$\begin{array}{c}62.5 \pm 10.8 \\
26-96 \\
64.5\end{array}$} & \multicolumn{2}{|c|}{$\begin{array}{c}66.0 \pm 10.4 \\
46-83 \\
65.5\end{array}$} & $\mathrm{U}=1.2$ & 0.227 \\
\hline $\begin{array}{c}\text { WHO } \\
\text { Histological type }\end{array}$ & $\begin{array}{l}\text { Infiltrating } \mathrm{UC}(\mathrm{no}=59) \\
\text { Noninvasive papillary } \mathrm{UC}(\mathrm{no}=1)\end{array}$ & $\begin{array}{c}45 \\
1\end{array}$ & $\begin{array}{c}76.3 \\
100\end{array}$ & $\begin{array}{c}14 \\
0\end{array}$ & $\begin{array}{c}23.7 \\
0\end{array}$ & $\mathrm{FE}=0.310$ & 0.578 \\
\hline Grade & $\begin{array}{l}\text { low }(\mathrm{no}=12) \\
\text { high }(\mathrm{no}=48)\end{array}$ & $\begin{array}{l}10 \\
36\end{array}$ & $\begin{array}{c}83.3 \\
75\end{array}$ & $\begin{array}{c}2 \\
12\end{array}$ & $\begin{array}{c}16.7 \\
25\end{array}$ & $\mathrm{FE}=0.373$ & 0.542 \\
\hline stage group & $\begin{array}{l}\text { early }(\text { no }=19) \\
\text { advanced }(n o=41)\end{array}$ & $\begin{array}{l}19 \\
27\end{array}$ & $\begin{array}{l}100 \\
65.9\end{array}$ & $\begin{array}{c}0 \\
14\end{array}$ & $\begin{array}{c}0 \\
34.1\end{array}$ & $\mathrm{FE}=8.5$ & $0.003 *$ \\
\hline $\begin{array}{l}\text { lymph node } \\
\quad(n o=41)\end{array}$ & $\begin{array}{l}\text { Absent }(n o=14) \\
\text { Present }(\text { no }=27)\end{array}$ & $\begin{array}{c}8 \\
19\end{array}$ & $\begin{array}{l}57.1 \\
70.4\end{array}$ & $\begin{array}{l}6 \\
8\end{array}$ & $\begin{array}{l}42.9 \\
29.6\end{array}$ & $\mathrm{FE}=9.3$ & $0.025^{*}$ \\
\hline Bilharzasis & $\begin{array}{l}\text { absent }(n o=41) \\
\text { present }(n o=19)\end{array}$ & $\begin{array}{l}34 \\
12\end{array}$ & $\begin{array}{l}82.9 \\
63.2\end{array}$ & $\begin{array}{l}7 \\
7\end{array}$ & $\begin{array}{l}17.1 \\
36.8\end{array}$ & $\mathrm{FE}=2.8$ & 0.092 \\
\hline LVI & $\begin{array}{l}\text { absent }(\mathrm{no}=43) \\
\text { present }(\mathrm{no}=17)\end{array}$ & $\begin{array}{c}38 \\
8\end{array}$ & $\begin{array}{l}88.4 \\
41.7\end{array}$ & $\begin{array}{l}5 \\
9\end{array}$ & $\begin{array}{l}11.6 \\
52.9\end{array}$ & $\mathrm{FE}=11.6$ & $0.001 *$ \\
\hline PNI & $\begin{array}{l}\text { absent }(\mathrm{no}=52) \\
\text { present }(\mathrm{no}=8)\end{array}$ & $\begin{array}{c}41 \\
5\end{array}$ & $\begin{array}{l}78.8 \\
62.5\end{array}$ & $\begin{array}{c}11 \\
3\end{array}$ & $\begin{array}{l}21.2 \\
37.5\end{array}$ & $\mathrm{FE}=1.03$ & 0.309 \\
\hline Necrosis & $\begin{array}{l}\text { absent }(\mathrm{no}=38) \\
\text { present }(\mathrm{no}=22)\end{array}$ & $\begin{array}{l}30 \\
16\end{array}$ & $\begin{array}{l}78.9 \\
72.7\end{array}$ & $\begin{array}{l}8 \\
6\end{array}$ & $\begin{array}{l}21.1 \\
27.3\end{array}$ & $\left(x^{2}\right)=0.301$ & 0.583 \\
\hline Stromal reaction & $\begin{array}{l}\text { Desmoplastic }(\mathrm{no}=25) \\
\text { inflammatory }(\mathrm{no}=26) \\
\text { both }(\mathrm{no}=9)\end{array}$ & $\begin{array}{c}22 \\
17 \\
7\end{array}$ & $\begin{array}{c}88 \\
65.4 \\
77.8\end{array}$ & $\begin{array}{l}3 \\
9 \\
2\end{array}$ & $\begin{array}{c}12 \\
34.6 \\
22.2\end{array}$ & $\mathrm{FE}=3.6$ & 0.161 \\
\hline Apoptosis & $\begin{array}{l}\text { mean } \pm \mathrm{SD} \\
\text { range } \\
\text { Median }\end{array}$ & \multicolumn{2}{|c|}{$\begin{array}{c}14.0 \pm 6.5 \\
3-31 \\
13.5\end{array}$} & \multicolumn{2}{|c|}{$\begin{array}{c}17.5 \pm 8.3 \\
8-31 \\
14.5\end{array}$} & $\mathrm{U}=1.22$ & 0.220 \\
\hline Mitosis & $\begin{array}{l}\text { mean } \pm \mathrm{SD} \\
\text { range } \\
\text { Median }\end{array}$ & \multicolumn{2}{|c|}{$\begin{array}{c}5.6 \pm 2.4 \\
2-15 \\
5\end{array}$} & \multicolumn{2}{|c|}{$\begin{array}{c}7.6 \pm 2.7 \\
4-15 \\
7\end{array}$} & $\mathrm{U}=2.6$ & $0.008 *$ \\
\hline
\end{tabular}

Cyclin D1 high expression in the current study is associated with the early-stage group $(P=0.031)$, in agreement with several studies (Galmozzi et al., 2006; Lenz et al., 2012; Amer and Eid, 2019; Lee et al., 2010) who stated low level of Cyclin D1 in the advanced stage, poorly differentiated tumors, vascular invasion, as well as lymph node involvement and MIBC.

In the current study, all high IRS Cyclin D1 cases (20/20) displayed absence of bilharzaial infestation ( $P=0.001)$ in agreement with a study done by (Zaghloul, 2012) who demonstrated that schistosomiasis associated UBC presented in more advanced stage than schistosomiasis non associated UBC supporting the favorable prognostic impact of Cyclin D1.

The favorable prognostic impact inferred by Cyclin D1 overexpression is attributed to its evidence in the initial stages where cell proliferation is a necessary step, involving no tumor invasion or metastasis as suggested by Guang and Tian (2015) and that low Cyclin D1 expression might be a surrogate of other genetic events in the same cells, which ultimately drives cell growth and leads to worse prognosis (Sud, 1998). Moreover, the phenotype of Cyclin D1 was correlated with the degree of cancer progression and invasiveness.

Altered expression of Cyclin D1 may lead to changes in the biological behavior of transformed cells, for instance, growth, proliferation, invasion and metastasis (Amer and Eid, 2019). The inverse correlation between Cyclin D1expression and poor prognostic parameters was not only reported in urothelial carcinoma; but also among other tumors, as in gastric carcinoma (Feakins et al., 2003), in laryngeal squamous cell carcinoma (Jovanovic et al., 2014) and invasive breast carcinoma (Parvin et al., 2019). 
Table 6. Relationship between peritumoral FOXP3 expression and different clinico-pathological parameters in malignant cases

\begin{tabular}{|c|c|c|c|c|c|c|c|}
\hline & \multirow[b]{2}{*}{ Variables } & \multicolumn{4}{|c|}{ Peritumoral FOXP3 expression } & \multirow{3}{*}{$\begin{array}{c}\text { Test of } \\
\text { significanc } \\
\mathrm{e} \\
\end{array}$} & \multirow[b]{2}{*}{$P$ value } \\
\hline & & \multicolumn{3}{|c|}{$\begin{array}{l}\text { positive } \\
(\text { no }=33)\end{array}$} & $\begin{array}{c}\text { Negative } \\
(n 0=47)\end{array}$ & & \\
\hline \multirow[b]{2}{*}{ Gender } & \multirow[b]{2}{*}{$\begin{array}{l}\text { Male }(n o=66) \\
\text { Female }(n o=14)\end{array}$} & $\mathrm{NO}$ & $\%$ & NO & $\%$ & & \multirow[b]{2}{*}{0.643} \\
\hline & & $\begin{array}{c}28 \\
5\end{array}$ & $\begin{array}{l}42.4 \\
35.7\end{array}$ & $\begin{array}{c}38 \\
9\end{array}$ & $\begin{array}{l}57.6 \\
64.3\end{array}$ & $\left(\mathrm{x}^{2}\right)=0.215$ & \\
\hline age/years & $\begin{array}{l}\text { mean } \pm \mathrm{SD} \\
\text { range } \\
\text { Median }\end{array}$ & \multicolumn{2}{|c|}{$\begin{array}{c}62.1 \pm 10.0 \\
26-80 \\
64\end{array}$} & \multicolumn{2}{|c|}{$\begin{array}{c}64.3 \pm 10.5 \\
42-96 \\
65\end{array}$} & $\mathrm{U}=0.387$ & 0.699 \\
\hline $\begin{array}{c}\text { WHO } \\
\text { Histological } \\
\text { type }\end{array}$ & $\begin{array}{l}\text { Infiltrating } \mathrm{UC}(\mathrm{no}=76) \\
\text { Noninvasive papillary } \mathrm{UC} \\
(\mathrm{no}=4)\end{array}$ & $\begin{array}{c}31 \\
2\end{array}$ & $\begin{array}{c}41.3 \\
50\end{array}$ & $\begin{array}{c}45 \\
2\end{array}$ & $\begin{array}{c}58.7 \\
50\end{array}$ & $\mathrm{FE}=0.829$ & 0.661 \\
\hline Grade & $\begin{array}{l}\text { Low }(n o=18) \\
\text { high }(n o=62)\end{array}$ & $\begin{array}{c}9 \\
24\end{array}$ & $\begin{array}{c}50 \\
38.7\end{array}$ & $\begin{array}{c}9 \\
38\end{array}$ & $\begin{array}{c}50 \\
61.3\end{array}$ & $\left(x^{2}\right)=0.734$ & 0.392 \\
\hline stage group & $\begin{array}{l}\text { early }(\mathrm{no}=30) \\
\text { advanced }(\mathrm{no}=50)\end{array}$ & $\begin{array}{l}12 \\
21\end{array}$ & $\begin{array}{l}40 \\
42\end{array}$ & $\begin{array}{l}18 \\
29\end{array}$ & $\begin{array}{l}60 \\
58\end{array}$ & $\left(x^{2}\right)=0.031$ & $0.001^{*}$ \\
\hline $\begin{array}{c}\text { lymph node } \\
(\text { no=50) }\end{array}$ & $\begin{array}{l}\text { Absent }(n o=20) \\
\text { Present }(n o=30)\end{array}$ & $\begin{array}{c}8 \\
13\end{array}$ & $\begin{array}{c}40 \\
43.3\end{array}$ & $\begin{array}{l}12 \\
17\end{array}$ & $\begin{array}{c}60 \\
65.7\end{array}$ & $\mathrm{FE}=0.118$ & 0.990 \\
\hline Bilharasis & $\begin{array}{l}\text { absent }(\mathrm{no}=56) \\
\text { present }(\mathrm{no}=24)\end{array}$ & $\begin{array}{l}21 \\
12\end{array}$ & $\begin{array}{c}37.5 \\
50\end{array}$ & $\begin{array}{l}35 \\
12\end{array}$ & $\begin{array}{c}62.5 \\
50\end{array}$ & $\mathrm{x}^{2}=1.1$ & 0.298 \\
\hline LVI & $\begin{array}{l}\text { absent }(\mathrm{no}=60) \\
\text { present }(\mathrm{no}=20)\end{array}$ & $\begin{array}{l}23 \\
10\end{array}$ & $\begin{array}{c}38.3 \\
50\end{array}$ & $\begin{array}{l}37 \\
10\end{array}$ & $\begin{array}{c}61.7 \\
50\end{array}$ & $\mathrm{x}^{2}=0.842$ & 0.359 \\
\hline PNI & $\begin{array}{l}\text { absent }(n o=70) \\
\text { present }(n o=10)\end{array}$ & $\begin{array}{c}28 \\
5\end{array}$ & $\begin{array}{l}40 \\
50\end{array}$ & $\begin{array}{c}42 \\
5\end{array}$ & $\begin{array}{l}60 \\
50\end{array}$ & $\mathrm{FE}=0.361$ & 0.548 \\
\hline Necrosis & $\begin{array}{l}\text { absent }(\mathrm{no}=52) \\
\text { present }(\mathrm{no}=28)\end{array}$ & $\begin{array}{c}24 \\
9\end{array}$ & $\begin{array}{l}46.2 \\
32.1\end{array}$ & $\begin{array}{l}28 \\
19\end{array}$ & $\begin{array}{l}53.8 \\
67.9\end{array}$ & $x^{2}=1.4$ & 0.225 \\
\hline $\begin{array}{l}\text { Stromal } \\
\text { reaction }\end{array}$ & $\begin{array}{l}\text { desmoplastic }(\mathrm{no}=38) \\
\text { inflammatory }(\mathrm{no}=29) \\
\text { both }(\mathrm{no}=13)\end{array}$ & $\begin{array}{c}1 \\
23 \\
9\end{array}$ & $\begin{array}{c}2.6 \\
79.3 \\
69.2\end{array}$ & $\begin{array}{c}37 \\
6 \\
4\end{array}$ & $\begin{array}{l}97.4 \\
20.7 \\
30.8\end{array}$ & $\mathrm{x}^{2}=44.9$ & $0.001^{*}$ \\
\hline Apoptosis & $\begin{array}{l}\text { mean } \pm \mathrm{SD} \\
\text { range } \\
\text { Median }\end{array}$ & & $\begin{array}{l} \pm 5.9 \\
31 \\
4\end{array}$ & & $\begin{array}{l}6 \pm 6.9 \\
-31 \\
14\end{array}$ & $\mathrm{U}=0.656$ & 0.512 \\
\hline Mitosis & $\begin{array}{l}\text { mean } \pm \mathrm{SD} \\
\text { range } \\
\text { Median }\end{array}$ & & $\begin{array}{l}2.4 \\
15 \\
5\end{array}$ & & $\begin{array}{l}2 \pm 2.7 \\
-15 \\
6\end{array}$ & $\mathrm{U}=1.35$ & 0.176 \\
\hline
\end{tabular}

Table 7. Correlation between peritumoral FOXP3 $\mathrm{H}$-score and tumoral FOXP3 $\mathrm{H}$-score

\begin{tabular}{|c|c|c|}
\hline Markers & \multicolumn{2}{c|}{ FOXP3 in tumor cells } \\
\cline { 2 - 3 } & $\mathrm{r}$ & P-value \\
\hline FOXP3 immunohistochemistry in peritumoral lymphocytes & 0.421 & $0.001^{*}$ \\
\hline
\end{tabular}

Regarding the expression of FOXP3 in the studied groups, FOXP3 was expressed only in the epithelium in the non-neoplastic group and was found to be expressed in both malignant cells and lymphocytes (peritumoral and intratumoral) in cases of the malignant group.

In this current study, FOXP3was expressed in $85 \%$ of the normal urothelium and $75 \%$ of the tumor cells in the malignant group $(P>0.05)$. This is in contrast to a study done by (Winerdal et al., 2011) and (Zhang $t$ al., 2016) who found that FOXP3 was highly expressed in cancer cells of UBC. Meanwhile, a study done by (Zuo et al., 2007) found that FOXP3 was expressed in normal breast and down-regulated in adjacent mammary cancer, the study clarified that
ErbB2and Skp2genes in breast cancer and c-Myc gene in prostate cancer are repressed causing predominant missense mutations in breast and prostate cancer patients suggesting the role of FOXP3 as a tumor suppressor gene (Zuo et al., 2007; Wang et al., 2009).

High FOXP3 IRS, in the current study, is in favor of advanced stage $(p=0.003)$, lymph node metastasis ( $p=0.025), L V I$ and high mitotic count $(P=0.008)$. Association of tumoral FOXP3 with poor prognostic factors agrees with a study done by (Zuo et al., 2007) which demonstrated the association of tumoral FOXP3 with lymphatic metastasis, advanced stage and high proliferative index ( $\mathrm{Ki}-67 \geq 14 \%)$ in cancer breast. 
The prognostic role of FOXP3 in tumor cells has been studied for many years. In vitro, FOXP3 represses the transcription of the HER2, SKP2, MYC, MMP2, and UPA genes and induces the expression of p21 and LATS2. Thus, inhibited cell growth, cell migration and cell invasion have been observed in cell lines derived from breast, prostate and ovarian cancers that overexpress FOXP3 (Peng et al., 2019, Nishikawa \& Sakaguchi, 2014).

This poor outcome in cancer may be due to variable mechanisms. Regulatory T cells inhibit many adaptive and innate immune cells, including CD4+ $T$ cells, CD8+ $T$ cells, dendritic cells, macrophages, and $B$ cells. It has been shown that Treg cells also inhibit NK cells in a TGF- $b$ dependent manner ( Ralainirina et al., 2007). Also, Treg cells can suppress immune responses of effector $T$ cells as well as other immune cells through direct cell-cell contactdependent mechanisms and release of various soluble factors ( Schmidt et al., 2012).

Another explanation, Tregs can induce immune tolerance and lead to tumor progression by the following mechanisms: secretion of immunosuppressive molecules such as transforming growth factor beta (TGF $\beta$ ), IL-10 and CCL22; directly cytolysis of NK cells and CD8+ cells; metabolic disruption; and promoting angiogenesis (Gupta et al., 2007; Ohara et al., 2009; Facciabene et al., 2012; Devaud et al., 2014). On the other hand, Tregs can inhibit tumor-promoting inflammation induced by bacteria infection, thereby contributing to an improved outcome (Ladoire et al., 2011).

Tumor infiltrating lymphocytes (TILs) may be classified into lymphocytes infiltrating the tumor cell islets (thus being in direct contact with tumor cells) called intra-epithelial (intratumoral) and TILs lymphocytes infiltrating the tumor stroma (peritumoral infiltrating lymphocytes) (Hornychova et al., 2008).

Sakaguchi et al., 2008 demonstrated that tumor infiltrating lymphocytes (TILS) are composed of different kinds of lymphocytes, including CD4+ and CD8+ T cells, Tregs, B cells, NK cells and NKT cells. Treg cells expressing the transcription factor FOXP3 are naturally present in the immune system and their dysfunction due to
FOXP3 gene mutation causes fatal autoimmune disease (Sakaguchi et al., 2008).

Most FOXP3+ Treg cells are CD4+ T cells that express CD25 can suppress the activation, proliferation and effector functions of a wide range of immune cells displaying a central role in the prevention of immune diseases (Sakaguchi et al., 2008).

In the current study, tumoral FOXP3 $\mathrm{H}$. Score was significantly associated with an increase in peritumoral positively stained FOXP3+ lymphocytes $\mathrm{H}$-score $(\mathrm{p}=0.001)$ and this agrees with a study done by (Allan \& Hafler 2006) who reported that a high infiltration of FOXP3+ lymphocytes was accompanied by FOXP3+ tumor expression in cancer breast. FOXP3 expressed in the malignant cells succeeded to recruit lymphocytes infiltration into tumor microenvironment (Allan \& Hafler, 2006; Takenaka et al., 2013). Regarding the overall survival, in the current study, the presence of bilharziasis, advanced pathological T stage and presence of lymph node invasion showed poor impact on patient's outcome. Furthermore, by multivariate COX- regression analysis, the pathological T stage was the most independent prognostic factor affecting patient's overall survival agreeing with (Moch et al., 2016).

On contrary with several studies found that Trop2 expression had been associated with poor survival in various cancers including; CRC (Xu and Gu et al., 2009; Fang et al., 2009; Sukunthankar et al., 2010), breast cancer (Huang et al., 2005), gastric cancer (Muhlmann et al., 2009; Zhao et al., 2016; Zhao et al., 2017) and cancer cervix (Liu et al., 2013), our study failed to reveal a significant association between the Trop2 immunoreactivity and patient's survival. On the other hand, studies as (Pak et al., 2012) found that Trop2 overexpression was associated with better survival in non-small cell lung cancer (NSCLC) in patients with adenocarcinoma and may be a better prognostic marker in advanced stage adenocarcinoma.

Similarly, our study failed to reveal a significant association between the Cyclin D1 immunoreactivity and patient's overall survival. This was similar to (Sgambato et al., 2002), meanwhile (Khabaz et al., 2016) reported that 
decreased expression of Cyclin D1 was associated with poor prognosis.

Furthermore, our study failed to reveal a significant association between the tumoral FOXP3 immunoreactivity and patient's survival. This was contrary to (Hinz et al., 2007; Zhang et al., 2016; Merlo et al., 2009; Peng et al., 2019) who found that increased expression of FOXP3 was associated unfavourable prognosis in pancreas, bladder and breast carcinoma respectively which is in contrast to (Ladoire et al., 2011; Ma et al, 2014) who demonstrated a positive correlation between tumoral FOXP3 and survival in patients with HER2+ tumours who have received neoadjuvant therapy and in gastric carcinoma respectively. Findings in the current study might be explained by the small sample size of the studied cases.

An inverse relationship between Cyclin D1 Hscore and both Trop2 and FOXP3 $\mathrm{H}$-score in primary bladder carcinoma cases, in the current study, was demonstrated, as Cyclin D1 served as a good prognostic marker, while, Trop2 and FOXP3was an unfavorable element of bladder cancer patients. However, no previous studies assessed this relationship in the malignant tumor, yet this inverse relationship could be explained as proposed by a study done by (Guerra et al., 2013) , that the phosphorylated forms of Cyclin D1are faster-migrating forms having a shorter half-life and that Trop2 decreases Cyclin D1 expression overall.Trop2 forms an oncogenic chimeric Cyclin D1- Trop2 protein, implicated in cell transformation in urinary bladder carcinoma which eventually consumes the Cyclin D1 causing the inverse relationship between both Trop2 and Cyclin D, is a proposed explanation for the inverse relationship in the current study.

\section{CONCLUSION}

Both Trop2 and FOXP3 imply poor prognostic impact and predicts tumor aggressiveness in bladder carcinoma in Egyptian patients while Cyclin D1 implies a favorable one. The inverse relation between Cyclin D1 and Trop2 proposes the consumption of Cyclin D1 by Trop2 as a ligand in the urinary bladder carcinogenesis in bladder carcinoma with high grade, advanced stage, high mitotic count and muscle invasion. Trop2, FOXP3 and Cyclin D1 are suggested to be promising candidate biomarkers that might serve as prognostic factors for the prediction of tumor behavior in bladder carcinoma in Egyptian patients. Further studies conducted on a larger scale to convey the role of Trop2 and FOXP3 antagonists as potential targeted therapeutic tools in UBC, is recommended.

\section{List of abbreviations}

BC: Bladder cancer

Tregs : regulatory T lymphocytes

AJCC : American Joint Committee on Cancer

AJCC-UICC: American Joint Committee on

Cancer-Union International Center Cancer

staging system

NMIBC: Non-muscle- invasive bladder cancer

MIBC: Muscle invasive bladder cancer

UC: Urothelial carcinoma

IHC: immunohistochemical

IRS : immunoreactive score

PNI: perineural invasion

TILs :Tumor infiltrating lymphocytes

\section{DECLARATIONS}

The ethics committee in the faculty of medicine Menoufia University approved the purpose of this retrospective study and the use of archival paraffin blocks to evaluate the expression of the studied primary antibodies.

\section{AVAILABILITY OF DATA AND MATERIALS}

The data that support the findings of this study are available from [third party name] but restrictions apply to the availability of these data, which were used under license for the current study, and so are not publicly available. Data are however available from the authors upon reasonable request and with permission of [third party name].

\section{CONFLICTS OF INTEREST}

All authors have approved this article and declare no conflicts of interest.

\section{FUND}

No fund was received for this work.

\section{References}

Ibrahim AS, Khaled HM, Mikhail NH, Baraka H, Kamel H. (2014). Cancer Incidence in Egypt: Results of the National Population-Based Cancer Registry Program. Journal of Cancer Epidemiology, 18:??-??. 
Heabah NA, Bedeer AE. (2021). Assessment of PD-L1 and p53 expression in urinary bladder carcinoma: Association with different clinicopathologic characteristics. International Journal of Cancer and Biomedical Research, 5(1):15-26.

Helal T., Salman M., Ezz-Elarab SS (2015). Malignant urinary bladder system tumors. In Helal T, Salman M, Ezz-Elarab SS (eds) pathology based cancer registry 2001-2010. Ain Shams Faculty of Medicine; $7^{\text {th }}$ ch: 63-72.

Kyritsi F., Loffredo C.A., Zheng Y.L., Philips G., Amr S. (2018). 'Urinary Bladder Cancer in Egypt: Are There Gender Differences in Its Histopathological Presentation?'. Advances in Urology, pp. 1-7.

Tang G., Tang Q., Jia L., Chen Y., Lin L., Kuai X., Gong A., Feng Z (2019). TROP2 increases growth and metastasis of human oral squamous cell carcinoma through activation of the PI3K/Akt signaling pathway. Int J Mol Med 44, 2161-2170.

Fong D., Moser P., Krammel C., Gostner J., Margreiter R., Mitterer M., Gastl G., Spizzo G. (2008a). High Expression Of Trop-2 Correlates With Poor Prognosis In Pancreatic Cancer. British Journal of Cancer 99, 1290-1295.

Guerra E., Trerotola M., Aloisi A., Tripaldi R., Vacca G., La Sorda R., Lattanzio R., Piantelli M., Alberti S. (2013). The TROP-2 Signalling Network In Cancer Growth. Oncogene 32, 1594-1600

Baldin V., Lukas J., Marcote M.J., Pagano M., Draetta G. (1993). "Cyclin D1 is a nuclear protein required for cell cycle progression in G1". Genes \& Development 7, 812-21

Huang J.W., Shiau C.W., Yang Y.T., Kulp S.K., Chen K.F., Brueggemeier R.W., Shapiro C.L., Chen C.S. (2005). Peroxisome proliferatoractivated receptor gamma-independent ablation of cyclin D1 by thiazolidinediones and their derivatives in breast cancer cells. Mol Pharmacol 67, 1342-8.

Cubas R., Li M., Chen C., Yao Q (2009). Trop 2: a possible therapeutic target for late stage epithelial carcinomas. Biochim Biophys Acta 1796, 309-314

Lopes J.E., Torgerson T.R., Schubert L.A., Anover S.D., Ocheltree E.L., Ochs H.D., Ziegler S.F. (2006). Analysis of FOXP3 reveals multiple domains required for its function as a transcriptional repressor. J Immunol 177, 3133-42.

Fontenot J.D., Gavin M.A., Rudensky A.Y. (2003). Foxp3 programs the development and function of $\mathrm{CD} 4+\mathrm{CD} 25+$ regulatory $\mathrm{T}$ cells. Nat. Immunol 4, 330-336
Martin F., Ladoire S., Mignot G., Apetoh L., Ghiringhelli F. (2010). Human FOXP3 and cancer. Oncogene 29, 4121-9.

Moch H., Humphrey P.A., Ulbright T.M., Reuter V. (2016). Tumor of urinary tract In: Moch $H$., Humphrey PA., Ulbright TM. and Reuter V. (eds), WHO Classification of Tumours of the Urinary System and Male Genital Organs. $4^{\text {th }}$ ED, Lyon, France: International Agency for Research on Cancer, $2^{\text {nd }} \mathrm{ch}, 78-133$.

Chauhan R, Verma N, Sharma SP, Bhargava R, Singh P. (2016). Role of apoptotic index, mitotic index and MIB-1 antibody expression as biomarkers in preneoplastic and neoplastic lesions of uterine cervix. Int J Res Med Sci. 4(6): 2093-100

Boustead G.B., Fowler S., Swamy R., Kocklebergh R., Hounsome L. (2014). Section of Oncology, BAUS (2014). Stage, grade and pathological characteristics of bladder cancer in the UK: British Association of Urological Surgeons (BAUS) urological tumour registry. BJU Int 113, 924-30.

Avellini C. , Licini C., Lazzarini R., Gesuita R., Guerra E., Tossetta G., Castellucci C., Giannubilo S.R., Procopio A., Alberti S., Mazzucchelli R., Olivieri F., Marzioni D. (2017). The trophoblast cell surface antigen 2 and miR$125 \mathrm{~b}$ axis in urothelial bladder cancer: Oncotarget 8, 58642-58653.

Amer A.I., Eid A.M. (2019). Prognostic significance of Cyclin D1 in urothelial carcinoma; correlation with p53 and clinicopathological parameters. J Am Sci 15, 86-91.

Winerdal M.E., Marits P., Winerdal M., Hasan M., Rosenblatt R., Tolf A., Selling K., Sherif A., Winqvist O. (2011). FOXP3 and survival in urinary bladder cancer. BJU Int 108, 1672-8

Smyth J., Gourley C., Walker G. (2007). Antiestrogen Therapy Is Active in Selected Ovarian Cancer Cases: The Use of Letrozole in Estrogen Receptor-Positive Patients. Clin Cancer Res 3617-3622.

Godlewski J., Kkieun J., Krazinski B.E., koielec Z., Wierzbicki P.M., Kmiec Z. (2018). The immunoexpression of YAP1 and LATS1 proteins in clear cell renal cell carcinoma: Impact on patients survival. Biomed Res Int. 2653623.

Dawson B, Trapp R (eds) (2001). Basic and clinical biostatistics: large medical books. Oxford, London. Boston.pp. 270-275.

Zhang L., Yang G., Jiang H., Liu M., Chen H., Huang Y., Wang Z. \& Bo J. (2017). Trop2 is associated with the recurrence of patients with nonmuscle invasive bladder cancer. Int J Clin Exp Med 10, 1643-1650. 
Ohmachi T., Tanaka F., Mimori K., Inoue H., Yanaga K., Mori M (2006). Clinical Significance Of TROP-2 Expression In Colorectal Cancer. Clinical Cancer Research 12, 3057-3063

Bignotti E., Todeschini P., Calza S., Falchetti M., Ravanini M., Tassi R.A., Ravaggi A., Bandiera E., Romani C., Zanotti L., Tognon G., Odicino F.E., Facchetti F., Pecorelli S. \& Santin A.D. (2010). Trop-2 overexpression as an independent marker for poor overall survival in ovarian carcinoma patients. Eur J Cancer 46, 944-953.

Stepan L., Trueblood E., Hale K., Babcook J., Borges L., Sutherland C. (2011). Expression Of TROP2 Cell Surface Glycoprotein In Normal And Tumor Tissues Potential Implications As A Cancer Therapeutic Target. Journal Of Histochemistry \& Cytochemistry 59, 701-710.

Zaman S., Jadid H., Denson A.C., Gray E (2019). Targeting Trop-2 in solid tumors: future prospects.Onco Targets Ther12, 1781-1790.

Shvartsur A., Bonavida B. (2015). Trop-2 And Its Overexpression In Cancers: Regulation And Clinical/Therapeutic Implications. Genes \& Cancer 6, 84-105.

Litvinov S.V., Balzar M., Winter M.J., Bakker H.A.M., Briaire-De Bruijn I.H., Prins F., Fleuren G.J., Warnaar S.O. (1997). Epithelial cell adhesion molecule (Ep-CAM) modulates cellcell interactions mediated by classic cadherins. J Cell Biol 139,1337-1348.

Balzar M., Briaire-De Bruijn I.H., Rees-Bakker H.A.M., Prins F.A., Helfrich W., De Leij L., Riethmuller G., Alberti S., Warnaar S.O., Fleuren G.J., Litvinov S.V. (2001). Epidermal growth factor-like repeats mediate lateral and reciprocal interactions of Ep-CAM molecules in homophilic adhesions. Mol Cell Biol 21, 2570-2580.

Li Z., Jiang X., Zhang W. (2016). Trop2 overexpression promotes proliferation and invasion of lung adenocarcinoma cells. Biochem Biophys Res Commun 470, 197-204.

Li X., Teng S., Zhang Y., Zhang W., Zhang X., Xu K., Yao H., Yao J., Wang H., Liang X., Hu Z. (2017). TROP2 promotes proliferation, migration and metastasis of gallbladder cancer cells by regulating $\mathrm{PI} 3 \mathrm{~K} / \mathrm{AKT}$ pathway and inducing EMT. Oncotarget 8, 47052-47063

Liu T., Liu Y., Bao X., Tian J., Yang X (2013). Overexpression of TROP2 predicts poor prognosis of patients with cervical cancer and promotes the proliferation and invasion of cervical cancer cells by regulating ERK signaling pathway. PLoS One 8, e75864.

Zhang K., Jones L., Lim S., Maher C.A., Adkins D., Lewis J., Kimple R.J., Fertig E.J., Chung C.H.,
Van Tine B.A., Ellis M.J., Herrlich A., Michel L.S. (2014). Loss of Trop2 causes ErbB3 activation through a neuregulin-1-dependent mechanism in the mesenchymal subtype of HNSCC. Oncotarget 5, 9281-9294

Redlich N., Robinson A.M., Nickel K.P., Stein A.P., Wheeler D.L., Adkins D.R., Uppaluri R., Kimple R.J., Tine B.A., Michel L.S. (2018). Anti-Trop2 blockade enhances the therapeutic efficacy of ErbB3 inhibition in head and neck squamous cell carcinoma. Cell Death Dis, 5.

Khabaz M.N., Buhmeida A., Ghabrah T., Qureshi I.A., Butt N.S., Al-Maghrabi B., Nedjadi T., AlQahtani M., Al-Maghrabi J. (2016). Cyclin D1 expression is associated with stage, grade and survival in urinary bladder carcinoma. Int J Clin Exp Med 9, 23482-23490

Shariat S.F., Ashfaq R., Sagalowsky A.I., Lotan Y. (2007). Association of cyclin D1 and E1 expression with disease progression and biomarkers in patients with non muscleinvasive urothelial cell carcinoma of the bladder. Urol Oncol 25, 468-75.

Kopparapu P.K., Boorjian S.A., Robinson B.D., Downes M., Gudas L.J., Mongan N.P., Persson J.L. (2013). Expression of Cyclin D1 and Its Association with Disease Characteristics in Bladder Cancer. Anticancer Res 33, 5235-5242.

Yan R., Wu X., Wang Y., Yu C., Li H., Zhang L. (2017). Overexpression of peptidyl-prolyl isomerase 1 (Pin1) and cyclin D1 in endometrial cancer Int J Clin Exp Pathol 10, 3335-3343.

Takagi Y., Takashi M., Koshikawa T., Sakata T., Ohshima S. (2000). Immunohistochemical demonstration of cyclin D1 in bladder cancers as an inverse indicator of invasiveness but not an independent prognostic factor. Int J Urol 7, 366-72.

Mhawech P., Greloz V., Oppikofer C., Szalay-Quinodoz I., Herrmann F. (2004). Expression of cell cycle proteins in T1a and T1b urothelial bladder carcinoma and their value in predicting tumor progression. Cancer 100, 2367-75.

Fristrup N., Birkenkamp-Demtroder K., Reinert T., Sanchez-Carbayo M., Segersten U., Malmstrom P.U., Palou J., Alvarez-Mugica M., Pan C.C., Ulhoi B.P., Borre M., Orntoft T.F., Dyrskjot L. (2014). Multicenter validation of cyclin D1, MCM7, TRIM29, and UBE2C as prognostic protein markers in non-muscleinvasive bladder cancer. Am J Pathol 182, 339-49.

Bahnassy A.A., Zekri A.R., El-Houssini S., ElShehaby A.M., Mahmoud M.R., Abdallah S., 
El-Serafi M. (2004). Cyclin A and cyclin D1 as significant prognostic markers in color ectal cancer patients. BMC Gastroenterol 23, 4:22.

Gao P., ZhouY., Liu Y., Li J.S., Zhen J.H., Yuan Y.P. (2004). Alteration of cyclin D1 in gastric carcinoma and its clinicopathologic significance. World J Gastroenterol 10, 29362939.

Shan Y.S., Hsu H.P., Lai M.D., Hung Y.H., Wang C.Y., Yen M.C., ChenY.L. (2017). Cyclin D1 overexpression correlates with poor tumor differentiation and prognosis in gastric cancer. Oncol Lett 14, 4517-4526.

Galmozzi F., Rubagotti A., Romagnoli A., Carmignani G., Perdelli L., Gatteschi B., F. (2006). Prognostic value of cell cycle regulatory proteins in muscle-infiltrating bladder cancer. J Cancer Res Clin Oncol 132, 757-64.

Lenz P., Pfeiffer R., Baris D., Schned A.R., Takikita M., Poscablo M.C., Schwenn M., Johnson A., Jones M., Kida M., Cantor K.P., Rothman N., Silverman D.T., Hewitt S.M., Moore L.E. (2012). Cell-cycle control in urothelial carcinoma. large-scale tissue array analysis of tumor tissue from Maine and Vermont. Cancer Epidemiol Biomarkers Prev 21, 155564.

Lee T.K., Miyamoto H., Osunkoya A.O., Guo C.C., Weiss S.W., Epstein J.I. (2010). Smooth muscle neoplasms of the urinary bladder: a clinicopathologic study of 51 cases. Am J Surg Pathol 34, 502-9.

Zaghloul M.S. (2012). Bladder cancer and schistosomiasis. Journal of Egyptian National Cancer Institute; No longer published by Elsevier, 151-159.

Guang S., Tian T. (2015). Expression of cyclin D1 and cyclin $E$ in urothelial bladder carcinoma detected in tissue chips using a quantum dot immunofluorescence technique. Oncol Lett 10, 1271-1276.

Sud N. (1998). Cloning of The Murine TROP-2 Gene: Conservation of A Pip2-Binding Sequence In The Cytoplasmic Domain Of TROP-2. Int. J. Cancer 75, 324-330.

Feakins R.M., Nickols C.D., Bidd H. \& Walton S.J. (2003) Abnormal expression of pRb, p16, and cyclin D1 in gastric adenocarcinoma and its lymph node metastases: relationship with pathological features and survival. Hum Pathol 34, 1276-82.

Jovanovic I.P., Radosavljevic G.D., SimovicMarkovic B.J., Stojanovic S.P., Stefanovic S.M., Peinovic N.N., Arsenijevic N.N. (2014). Clinical significance of Cyclin D1, FGF3 and p21 protein expression in laryngeal squamous cell carcinoma. J BUON 19, 944-52.

Parvin T., Das C., Choudhury M., Chattopadhyay B.K., Mukhopadhyay M. (2019). prognostic utility of Cyclin D1 in invasive breast carcinoma. Indian J Surg Oncol 10, 167-173

Zhang H., Prado K., Zhang K.X., Peek E.M., Lee J., Wang X., Huang J., Li G., Matteo Pellegrini M., Chin A.I. (2016). Biased Expression of theFOXP3 $\Delta$ 3Isoform in Aggressive Bladder Cancer Mediates Differentiation and Cisplatin Chemotherapy Resistance. Clin Cancer Res 22, 5349-5361.

Zuo T., Liu R., Zhang H., Chang X., Liu Y., Wang L., Zheng P., Liu Y. (2007). FOXP3 is a novel transcriptional repressor for the breast cancer oncogene SKP2. J Clin Invest 117, 3765-73.

Wang L., Liu R., Li W., Chen C., Katoh H., Chen G.Y., Mcnally B., Lin L., Zhou P., Zuo T., Conney K.A., liu Y., Zheng P. (2009). Somatic Single Hits Inactivatethe X-Linked Tumor Suppressor FOXP3 in the Prostate. Cancer Cell 16, 33646.

Peng G.L., Li L., Guo Y.W., Yu P., Yin X.J., Wang S., Liu C.P. (2019). CD8+ cytotoxic and FoxP3+ regulatory $\mathrm{T}$ lymphocytes serve as prognostic factors in breast cancer. Am J Transl Res 11,5039-5053.

Nishikawa H., Sakaguchi S. (2014). Regulatory T cells in cancer immunotherapy. Current opinion in immunology. Curr Op in Immunol 27, 1-7.

Ralainirina N., Poli A., Michel T., Poos L., Andres E., Hentges F., Zimmer J (2007). Control of NK cell functions by CD4+CD25+ regulatory T cells. J Leukoc Biol 81, 144-153.

Schmidt A., Oberle N., Krammer P.H. (2012). Molecular Mechanisms of Treg-Mediated $\mathrm{T}$ Cell Suppression. Front Immunol 3, 51.

Gupta S., Joshi K., Wig J.D., Arora S.K. (2007). Intratumoral FOXP3 expression in infiltrating breast carcinoma: Its association with clinicopathologic parameters and angiogenesis. Acta Oncologica 46, 792-797

Ohara M., Yamaguchi Y., Matsuura K., Murakami S., Arihiro K., Okada M. (2009). Possible involvement of regulatory $\mathrm{T}$ cells in tumor onset and progression in primary breast cancer. Cancer Immunol Immunother 58, 441-447.

Facciabene A., Motz G.T., Coukos G. (2012). Tregulatory cells: key players in tumor immune escape and angiogenesis. Cancer research 72, 2162-2171.

Devaud C., Darcy P.K., Kershaw M.H. (2014). Foxp3 expression in T regulatory cells and other cell 
lineages. Cancer immunology, immunotherapy: Cll 63, 869-876.

Ladoire S., Martin F., Ghiringhelli F. (2011). Prognostic role of FOXP3+ regulatory $T$ cells infiltrating human carcinomas: the paradox of colorectal cancer. Cancer immunology, immunotherapy: CII. 60(7): 909-18.

Hornychova H., Melichar B., Tomsova M., Mergancova J., Urminska H., Ryska A. (2008). Tumor-infiltrating lymphocytes predict response to neoadjuvant chemotherapy in patients with breast carcinoma. Cancer Invest 26, 1024-1031.

Sakaguchi S., Yamaguchi T., Nomura T., Ono M. (2008). Regulatory $T$ cells and immune tolerance. Cell 133, 775-787.

Allan C.B., Hafler D.A. (2006). Human regulatory T cells and their role in autoimmune disease. Immunol. Rev 212, 203-216.

Takenaka M., Seki N., Toh U., Hattori S., Kawahara A., Yamaguchi T., Koura K., Takahashi R., Otsuka H., Takahashi H., Iwakuma N., Nakagawa S., Fujii T., Sasada T., Yamaguchi R., Yano H., Shirouzu K., Kage M. (2013). FOXP3 expression in tumor cells and tumorinfiltrating lymphocytes is associated with breast cancer prognosis. Mol Clin Oncol 1, 625-632.

Xu K.Y., Gu J (2009). Expression of Trop2 mRNA in left-sided and right sided colon cancer and its clinical significance. Zhonghua Wei Chang Wai Ke Za Zhi 12, 285-289.

Fang Y.J., Lu Z.H., Wang G.Q., Pan Z.Z., Zhou Z.W., Yun J.P., Zhang M.F., Wan D.S. (2009). Elevated expressions of MMP7, Trop2, and surviving are associated with survival, disease recurrence, and liver metastasis of colon cancer. Int J Colorectal Dis 24, 875-884.

Sukunthankar M., Alberti S., Baek S.J. (2010). Epigallocatechin3-gallate (EGCG) posttranscriptionally and posttranslationally suppresses the cell proliferative protein Trop2 in human colorectal cancer cells. Anticancer Research 30, 2497-2503.

Muhlmann G., Spizzo G., Gostner J., Zitt M., Maie H., Moser P., Gastl G., Zitt M., Muller H.M., Margreiter R., Ofner D., Fong D (2009). Trop2 expression as prognostic marker for gastric carcinoma. J Clin Pathol 62, 152-158.
Zhao M., He X.L., Teng X.D. (2016). Understanding the molecular pathogenesis and prognostics of bladder cancer: an overview. Chin J Cancer Res 28, 92-8.

Zhao W., Ding G., Wen J., Tang Q., Yong H., Zhu H., Zhang S., Qiu Z., Feng Z., Zhu J. (2017). Correlation between Trop2 and amphiregulin coexpression and overall survival in gastric cancer. Cancer Med 6, 994-1001

Pak M.G., Shin D.H., Lee C.H., Lee M.K. (2012). Significance of EpCam and Trop2 expression in non-small cell lung cancer. World J Surg Oncol 10, 53.

Sgambato A., Migaldi M., Faraglia B., Aloysio G.D.E., Ferrari P., Ardito R., Gaetani C.D.E., Capelli G., Cittadinil A., Trentini G.P. (2002), Cyclin D1 expression in papillary superficial bladder cancer: its association with other cell cycle- associated proteins, cell proliferation and clinical outcome. Int. J. Cancer 97, 671678.

Hinz S., Pagerols-Raluy L., Oberg H.H., Ammerpohl O., Grussel S., Sipos B., Grutzmann R., Pilarsky C., Ungefroren H., Saeger H.D., Kloppel G., Kabelitz D., Kalthoff H. (2007). Foxp3 expression in pancreatic carcinoma cells as a novel mechanism of immune evasion in cancer. Cancer Res 67, 8344-8350.

Merlo A., Casalini P., Carcangiu M.L., Malventano C., Triulzi T., Mènard S., Tagliabue E., Balsari A. (2009). FOXP3 expression and overall survival in breast cancer. J Clin Oncol 27, 1746-1752.

Ma G.F., Miao Q., Liu Y.M., Gao H., Lian J.J., Wang Y.N., Zeng X.Q., Luo T.C., Ma L.L., Shen Z.B., Sun Y.H., Chen S.Y. (2014). High FoxP3 expression in tumour cells predicts better survival in gastric cancer and its role in tumour microenvironment. $\mathrm{Br} \mathrm{J}$ Cancer 110, 1552-1560. 